\title{
Connecting the D.O.Ts
}

\author{
Armand Krikorian
}

Published online: 22 February 2015

(c) Springer Science+Business Media New York 2015

Diabetes, Obesity and bone Trabeculae. Three entities with often unrecognized, or forgotten, connections. Nearly every article about diabetes nowadays mentions its staggering prevalence and impact on public health and cost. Similarly, obesity has been described as an 'epidemic', a rampant, widespread occurrence that is actually more akin to a pandemic with far-reaching impacts on human health. Such is the concern that some forecasts for life expectancy have projected shorter lifespans for today's children compared to their parents. With aging comes osteoporosis, a condition affecting so many individuals above the age of 50 that not being affected by this condition is slowly becoming the exception rather than the rule. The NIH 2014 actual budget expenditure for these three entities combined was 2 billion dollars, with minimal increases projected going into 2016 . While most agree that diabetes and obesity share some level of common pathophysiology, especially when considering type $2 \mathrm{DM}$, the articles in this issue ambitiously set to explore the different ways the three disease entities, diabetes, obesity and osteoporosis, are interrelated.

At a molecular level, the articles of Dr. Mazhari et al. and Dr. Gaualillo et al. explore the impact of various cytokines on bone health and glucose regulation and suggest several translational opportunities. Drs. Chaiban and Nicolas discuss the complex interactions between diabetes and bone health, with an emphasis on their bi-directional nature.

On the purely clinical side, we realize that our ability to treat osteoporosis is dependent on our ability to correctly identify it. Dr. Drever and her group review the various diagnostic modalities for osteoporosis and highlight the need for alternatives to bone mineral density in the special population of patients with diabetes and/or obesity. As we all share the excitement of discovery and readiness for clinical use of novel therapeutic agents for diabetes, the recent articles highlighting the potential negative effects of drugs on heart health serve to reminds us of our first pledge to 'do no harm'. Hence, Dr. Maalouf's article in this issue reviews in depth the available evidence, or lack of, regarding the impact of these agents on bone.

Diabetes, obesity and bone health need to be better connected. This issue serves as a reminder of the old adage that the more we know, the more we find out we don't know.

\footnotetext{
A. Krikorian $(\bowtie)$

Internal Medicine, UIC/Advocate Christ Medical Center,

4440 W. 95th Street, Oak Lawn, IL 60453, USA

e-mail: Armand.Krikorian@advocatehealth.com
} 